\title{
SURFACE ENERGY FROM ORDER PARAMETER PROFILE AT THE QCD PHASE TRANSITION
}

\author{
Z. Frei \\ Eötvös University, Budapest, Hungary \\ A. Patkós* \\ NASA/Fermilab Astrophysics Center \\ Fermi National Acceleratory Laboratory \\ Box 500, Batavia IL 60510-0500
}

Abstract: The order parameter profile between coexisting confined and plasma regions at the QCD phase transition is constructed. The dimensionless combination of the surface energy $(\sigma)$ and the correlation length $(\xi)$ is estimated to be

$$
\sigma \xi^{3} \approx 0.8 \text {. }
$$

\footnotetext{
(AASA-CR-184890) SURFACE EEEEGY FBCE CADER
EARAHETEE FRCFILE: AT THE CCE FEASE

TEANSITICN

lEOEt DCES

Icrand toiv.

\section{$7 \mathrm{p}$ \\ $\operatorname{CscI} 20 \mathrm{H}$}

$899-21633$

Onclas

G3/72 0199114
}

* Permanent address: Department of Atomic Physics, Eötrös University ,Budapest,Hungary 
The impact of the transition from the quark-gluon plasma to the hadronic phase on the baryon density fluctuations and primordial nucleosynthesis in the Early Universe is being actively investigated [1-4]. Quantitative description of the hadronic bubble formation, their percolation and the squeeze of the plasma droplets rely on data originating from quantum chromodynamics. The progress in the lattice measurements of the transition temperature and the latent heat is continuous and at present a rather weakly first order transition is suggested to take place $[5,6]$.

The average size, the rate of production etc. of hadronic bubbles are sensitive to a third quantity, the surface energy associated with the interface between coexisting confined and deconfined regions at the transition temperature. Very recently attempts have been made for numerical determination of this quantity directly in QCD $[7,8]$. The problem consists of calculating the excess free energy of the gluonic medium at $T_{c}$ resulting from the imposition of two parallel, infinitely distant walls of temperature $T=\infty$ and $T=0$, respectively. The exploratory Monte Carlo study [8] does not correspond yet to this ideal setup.

In this note we propose as an approximate realisation of the above strategy the evaluation of the surface energy in the framework of the mean field approximation to the order parameter (Polyakov-loop) theory. The strategy is to construct and quantitatively characterize the so called order parameter profile [9]. This approximation is widely used in various fields of statistical physics e.g. nucleation theory, wetting etc. The explicit form of the effective theory of Polyakov-loops we shall use is valid at strong gauge coupling only, still we believe that predictions on combinations of observables which are independent of the lattice spacing might be of more general validity as an order of magnitude estimate. (This expectation is based on the experience gained with strong coupling spectrum calculations [10]).

The derivation of the effective lattice theory of Polyakov-loops should be considered as a constrained integration in the partition function of the full, finite temperature gauge system. Were the integrations performed exactly, the "integrated out" contributions to the free energy kept, then no thermodynamic information should be lost. Furthermore the pieces independent of the Polyakov loop variables cannot produce the necessary singularities, therefore the latent heat, the order parameter discontinuity etc. should not depend on them.

To the present date, the integration has been performed only in the strong coupling regime $[11,12]$ :

$$
S_{e f f}=J_{e f f} \sum_{x, e}\left(\operatorname{Tr} U_{x} \operatorname{Tr} U_{z+e}^{+}+c . c .\right)
$$

where

$$
J_{e f f}=\left(\frac{1}{3 g^{2}}\right)^{1 / T a}
$$

( $g^{2}$ is the gauge coupling $\gg 1, T$ the temperature and $a$ the lattice constant in the temperature direction). We consider the pure $S U(3)$ gluon theory. Closer to 


\section{ORIGINAL PAGE IS \\ OF POOR QUALITY}

the continuum limit $\left(g^{2} \rightarrow 0, a \rightarrow 0\right)$ more complicated terms do appear in (1), with further $J$-type couplings depending on $g^{2}$ and $T$ nontrivially. For individual physical quantities there is no hope to see the continuum behavior. The best, the predictions for $a$-independent ratios can be taken seriously.

Let us denote the direction orthogonal to the boundary walls as the $x$-axis. The physical situation suggests to choose an $x$-dependent mean field $m(x)$ to minimize the approximate free energy per unit area given by the expression:

$$
F_{M . F .}=-\sum_{z} \ln \int d U \exp \left\{J M(x)\left(\operatorname{Tr} U_{z}+\operatorname{Tr} U_{z}^{+}\right)\right\}+J \sum_{x} m(x) M(x),
$$

where $M(x)=4 m(x)+m(x+1)+m(x-1)$ and $d U$ is the Haar-measure of the $S U(3)$ - invariant integration. One has to solve the selfconsistent equation:

$$
\frac{\delta F_{M . F .}}{\delta m(x)}=0
$$

subject to the boundary conditions

$$
m(x \rightarrow-\infty)=0, \quad m(x \rightarrow \infty)=m_{0} .
$$

The parameter $m_{0}$ is selected by the requirement of the existence of a soliton solution to eq.(4), smoothly extrapolating between the ordered and disordered phases (see below). Eq.(4) is understood as a local relationship between $M$ and $m$, or introducing the notation

$$
\begin{gathered}
m(x+1)=m(x)+i(x), \\
M(x)=6 m(x)+i(x)-i(x-1) \equiv 6 m(x)+I(x)
\end{gathered}
$$

a relation between $I$ and $m$. One realizes that $I(x)$ approximates $d^{2} m / d x^{2}$, therefore eq.(4) is actually the discrete version of a secorid order nonlinear differential equation. (For instance, in the 3-state Potts model this equation can be written in fully explicit form. With this identification the smoothness of the interpolation between the prescribed boundary values is naturally expressed by the boundary conditions:

$$
\frac{d m}{d x} \rightarrow 0, \quad \frac{d^{2} m}{d x^{2}} \rightarrow 0, \quad \text { if } \quad|x| \rightarrow \infty .
$$

On the lattice these conditions are equivalent to:

$$
\begin{gathered}
\int_{0}^{m_{0}} I(m) d m=0, \\
I(x) \rightarrow 0, \quad \text { if } \quad|x| \rightarrow \infty .
\end{gathered}
$$


What are the consequences of $(7 b)$ on the parameters $m_{0}$ and $J$ ? This question is answered starting from eq.(4), whose explicit form is:

$$
\frac{1}{2 J} \frac{d}{d M} \ln \int d U \exp \left\{J M\left(\operatorname{Tr} U+\operatorname{Tr} U^{+}\right)\right\}=m .
$$

Integration of eq.(8) with respect to $M$ on the interval $\left(0, M\left(m_{0}\right)\right)$ results in the following equality:

$$
\begin{gathered}
\ln \int d U \exp \left\{J M\left(m_{0}\right)\left(\operatorname{Tr} U+\operatorname{Tr} U^{+}\right)\right\}-J m_{0} M\left(m_{0}\right)- \\
-m_{0} I\left(m_{0}\right)-\int_{0}^{m_{0}} d m I(m)=0 .
\end{gathered}
$$

In view of (7b) the last two terms on the : hand side of (9) vanish. In this way eqs.(9),(8) are just the equations wh: etermine the transition point $J_{c}$ and the order parameter discontinuity $\left(m_{c}\right)$ in the conventional treatment of first order transitions.

The meaning of this result is that eq.(8) has a smoothly interpolating solution only at $J=J_{c}$ with $m_{0}=m_{c}$. Clearly, the physical statement of phase coexistence is equivalent to the existence of a solitonic order parameter profile.

The numerical construction of the kink solution starts by solving eqs.(7b),(8), (9) with $m(x) \equiv m_{c}$, which reproduces the well-known $J_{c}=0.134270$ and $m_{c}=$ 1.45786 data [9]. Using these values in (8), $m(x+1)$ is calculated from the knowledge of $m(x-1)$ and $m(x)$. The "initial" values were chosen to be $m(0)=0, m(1) \approx$ $o\left(10^{-10}\right)$. Each step of calculating the soliton in a new point involves two integrals to be done numerically. The accumulation of numerical errors might spoil the soliton.(The inaccurately calculated $m(x)$ function would reach $m_{0}=m_{c}$ with nonzero derivative, making the kink unstable). Therefore $J_{c}, m_{c}$ and the integrals require careful evaluation. (In case of the 3-state Potts model, the homogeneous mean field problem can be solved analytically and the sensitivity to deviations from $J_{c}$ and $m_{c}$ be studied in a systematic way). The kink constructed numerically is displayed in Fig.1.

The soliton can be characterized by two physical quantity. Its half-width provides an estimate of the order parameter correlation length at the transition. From Fig.1 one reads off $\xi \approx 2$ in lattice units. Substituting $m(x)$ into eq.(3) the surface energy in lattice units is

$$
\sigma=0.106 \text {. }
$$

A combination which is independent of the actual lattice spacing can be formed as

$$
\sigma \xi^{3} \approx 0.848 \text {. }
$$

This is the main message of our note, strictly speaking valid in the strong coupling regime. 


\section{ORIGINAL PAGE IS \\ OF POOR QUALTY}

However we cannot resist to conjecture a value of $\sigma$ in physical units extending the validity of (11) to weaker couplings. For this we use data from the most recent Monte Carlo investigations [5,6]. In Ref. [5] a detailed analysis of the order parameter correlation length was given. For "temporal size" $N_{t}=4$ two different spatial sizes led to $\xi \approx 9$ and $\xi \approx 13$, respectively at the transition point. Assuming that this system $\left(\beta_{c}=5.69\right)$ already obeys continuum physics one deduces

$$
\xi_{p h y}, T_{c} \approx 2.75 \pm 0.5 \text {. }
$$

Combining this with our result (11) one gets

$$
\sigma_{\text {phys }} \approx(0.04 \pm 0.02) T_{c}^{3} \text {. }
$$

Our result for $\sigma_{p h y s} / T_{c}^{3}$ is almost two orders of magnitude smaller, than that of Ref. [8]. We consider this shift towards smaller coefficients physically reasonable as one deals with a weakly first order transition (in continuous transitions $\sigma\left(T_{c}\right)=0$ ). Assuming $T_{c}=100 \mathrm{MeV}$ the range of $\sigma_{\text {phye }}$ from (13) practically coincides with the values considered by Fuller et al. [3] for astrophysical applications. For $T_{c}=$ $150 \mathrm{MeV}$ the values from (13) are at the higher end of the range considered in [3].

\section{Acknowledgements}

We are particularly grateful to Poul Damgaard for sharing with us his interest and scepticism on the subject. We have benefited from discussions with G. Fuller, J.Hertz, Z. Rácz and M. Turner. This research has been supported by a joint NSF-Hungarian Academy of Sciences grant. A.P. thanks the hospitality of the Astrophysics Group at FNAL where he was supported in part by NASA grant NAGW-1340.

\section{Figure Caption}

Fig. 1 The order parameter profile from the effective $S U(3)$ Polyakov-loop theory at the transition point. The correlation length $\xi$ can be read off directly. 


\section{ORIGINAL PAGE IS \\ OF POOR QUALITY}

\section{References}

[1] E. Witten, Phys. Rev. D30 (1984) 272

[2] J.H. Applegate, C.J. Hogan and R.J. Scherrer, Phys. Rev. D35 (1987) 1151

[3] G.M. Fuller, G.J. Mathews and C.R. Alcock, Phys. Rev. D37 (1987) 1380

[4] H. Kurki-Suonio et al., Phys. Rev. D (to appear)

[5] P. Bacilieri et al, Phys. Rev. Lett. 61 (1988) 1545

[6] F.R. Brown et al., Phys. Rev. Lett. 61 (1985) 2058

[7] C. Rebbi, Talk given at the Lattice ' 88 Conference (to appear in Nucl. Phys.)

[8] K. Kajantie and L. Kärkkäinen, Helsinki preprint HU-TFT-88-22

[9] J.S. Langer, Ann. Phys. 41 (1967)108

for review see:

K. Binder, Recent Progress in the Theory of First Order Phase Transitions: Statics and Dynamics (1986) Mainz-preprint

[10] N. Kawamoto and J. Smit, Nucl. Phys. 192 (1982) 100

H. Kluberg-Stern, A. Morel and B. Petersson, Nucl. Phys. B215 [FS7] (1983) 527

[11] J. Polónyi and K. Szlachányi, Phys. Lett. 110B (1982) 395

[12] F. Green and F. Karsch, Nucl. Phys. B238 (1984) 297 
ORIGINAL PAGE IS

OF POOR QUALITY

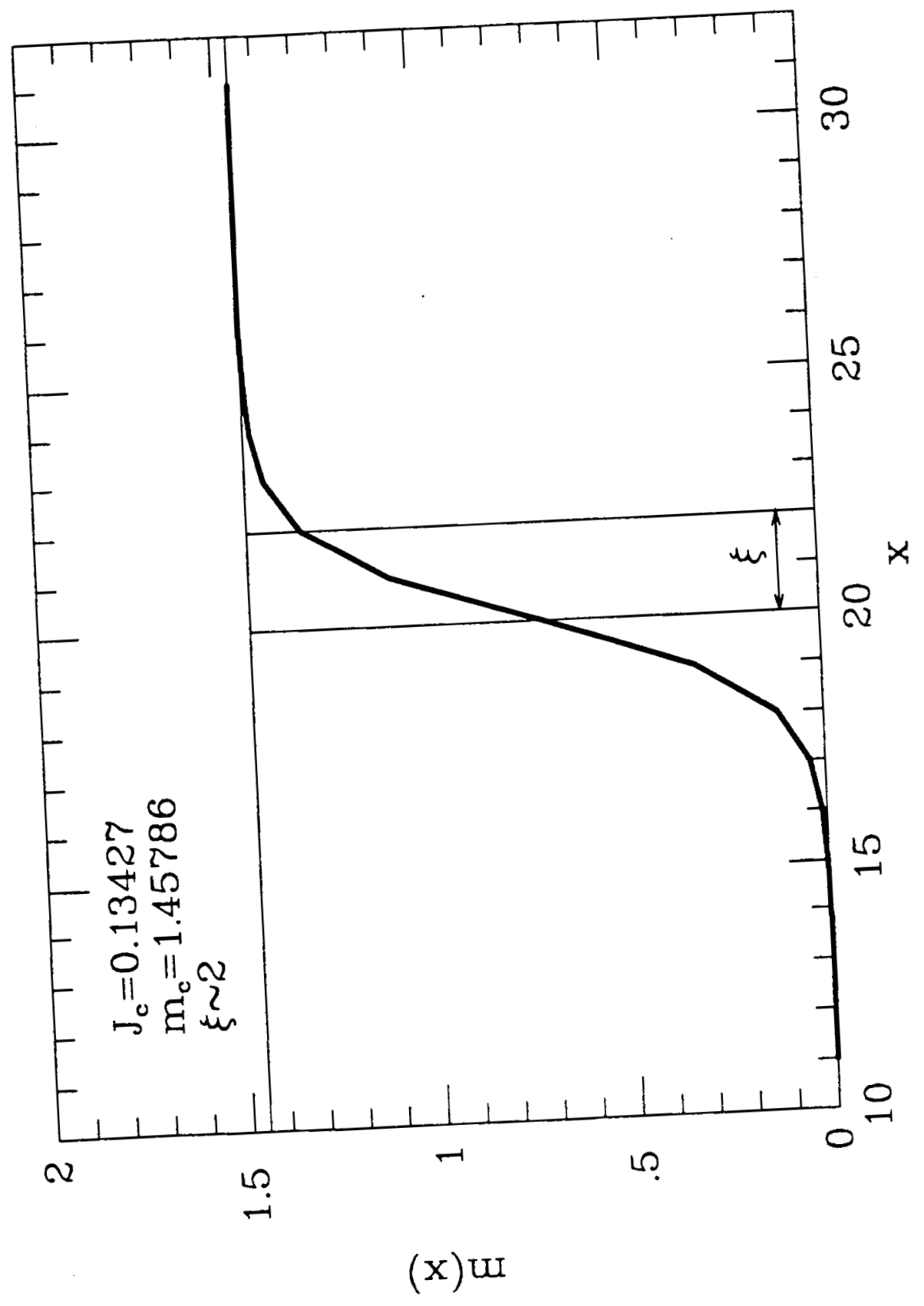

